\title{
Editorial
}

Rev. Enf. Neurol. (Mex)

\section{COORDINACIÓN CLÍNICA; AVANCE Y ESTRATEGIAS EN LA CALIDAD}

El profesional de enfermería hoy en día se enfrenta con un mundo globalizado donde la ciencia, la tecnología, el trabajo en equipo, el uso de modelos teóricos, el conocimiento de leyes, normas, reglamentos, procesos e indicadores entre otros de emisión nacional e internacional la orillan a perfeccionar sus procesos de trabajo; con la finalidad no sólo de proporcionar, sino de garantizar cuidados de alta calidad en cada uno de los escenarios en los que atiende a las personas, familias y comunidades y que éstos "indicadores" al emitir una evaluación en la satisfacción, nos de elementos donde se refleje el binomio cuidado-satisfacción objetivo primordial del sector de cuidados en un proceso de mejora continua.

La enfermería neurológica desde sus inicios en los años $80^{\prime}$ con su precursora Rosa María Casares Ayala, quien con una visión futurista creó el marco de acción de esta profesión en el área de las neurociencias, en una institución de tercer nivel dado la oportunidad a la conformación cualitativa y cuantitativa de capital humano, mismas que se han posicionado con paso firme en los diferentes entornos vistos desde la administración, enseñanza, investigación y la asistencia en esta última destacando dentro y fuera de nuestra institución.

En el marco de la calidad se crea en marzo del año 2007 la coordinación clínica dentro de una nueva propuesta de gestión de enfermería al ser el objetivo primordial el:

\section{"Garantizar en todo momento el cuidado enfermero de la más alta calidad a cada una de las personas atendidas en los diferentes servicios del instituto"}

Y para lograrlo se han llevado a cabo de diversas actividades y funciones de manera conjunta con el profesional enfermero, inicialmente se realizó una reingeniería del manual de procedimientos, conteniendo cada uno de los instrumentos de enfermería, tanto de atención directa como de control administrativo; a partir del monitoreo, evaluación y comisión de acciones de mejora de indicadores de enfermería, en beneficio directo del usuario, misma que dan pauta al desarrollo de guías clínicas y a el diseño e implementación del catálogo institucional de planes de cuidados, lo cual continuidad al transformación al modelo de supervisión, mediante la adopción y adaptación de estrategias y directrices que sustenten el hacer enfermero desde la perspectiva del líder, sustentando éste hacer en normas y procesos que se efectúan tanto a nivel nacionales como internacional.

Así mismo la incursión como representante del gremio en los diferentes comités, como son lo cual establece un nuevo modelo de colaboración institucional

De la misma forma la adecuación de equipamiento y funcionalidad de los equipos inicialmente el de carro de paro, derivado de ello la innovadora en la creación y funcionalidad del código verde, así como la adherencia colaboración del personal de salud a los diferentes programas que de manera continua propicie y den pauta a elevar y sustentar la calidad de atención de enfermería a través de la creación y análisis de encuestas de opinión.

Por ello la coordinación clínica fundamenta su existencia en los conocimientos sólidos y actualizadas de las ciencias humanistas y sustentando su proceder ante el cambiante avance tecnológico con el único fin de unificar criterios a bien de dar un cuidado integral de salud a las personas que más lo necesitan nuestros pacientes.

Mtra. Patricia Zamora Ruíz, Coordinadora Clínica

Instituto Nacional de Neurología y Neurocirugía

Manuel Velasco Suárez

patizaru@yahoo.com.mx

Enf Neurol Vol. 15. No. 1 enero - abril 2016 